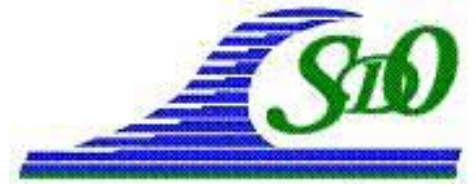

\title{
Variabilité du climat de houle dans le Golfe de Gascogne au cours des six dernières décennies
}

\author{
Xavier BERTIN $^{1,2}$, Guillaume DODET ${ }^{2}$
}

1. Centre National de la Recherche Scientifique, UMR LIENSS 6250, Institut du

Littoral et de l'Environnement, 2 rue Olympe de Gouges, 17000 La Rochelle, France. xbertin@univ-lr.fr

2. Division des Estuaires et Zones Côtières, Laboratoire National d'Ingénierie Civile, Av. do Brasil, 101, Lisbonne 1700-066, Portugal.

\section{Résumé :}

Cette étude présente les résultats d'une simulation rétrospective des vagues entre 1953 et 2009 basée sur le modèle régional de l'Atlantique Nord de DODET et al. (2010), dans lequel a été emboîtée une grille de résolution $0.2^{\circ}$ couvrant le Golfe de Gascogne et la partie occidentale de la Manche. Les hauteurs significatives $H_{s}$, périodes de pic $T_{p}$ et directions moyennes $D_{\text {ir }}$ montrent de fortes variabilités interannuelles, particulièrement lorsque les moyennes hivernales sont considérées. L'analyse par régression linéaire des séries temporelles de ces paramètres entre 1953 et 2009 montre : (1) une augmentation des hauteurs significatives qui atteint $+0.01 \mathrm{~m}^{\mathrm{an}}{ }^{-1}$ au nord de la zone d'étude ; (2) une augmentation des périodes de pic allant jusqu'à $+0.015{\mathrm{~s} . a n^{-1}}^{\mathrm{au}}$ sud du Golfe de Gascogne. La comparaison des séries temporelles de ces paramètres avec les moyennes hivernales de l'indice de l'oscillation nord Atlantique (NAO) révèle : (1) une corrélation positive entre l'indice $\mathrm{NAO}$ et $\mathrm{H}_{\mathrm{s}}$, qui augmente du sud au nord de la zone d'étude; (2) une corrélation positive entre l'indice NAO et Tp, qui augmente également du sud au nord et (3) une faible corrélation entre l'indice NAO et $\mathrm{D}_{\text {ir. }}$ Les tendances à long terme des paramètres des vagues sont expliquées par une augmentation de l'indice NAO au cours de la seconde moitié du XX ${ }^{\mathrm{ème}}$ siècle.

\section{Mots-clés :}

Climat de houle - Etats de mer - Modélisation numérique - Oscillation nord-atlantique

\section{Abstract:}

This study presents results obtained from a wave hindcast between 1953 and 2009, based on the regional model of the North Atlantic Ocean of Dodet et al., (2010), where a $0.2^{\circ}$ resolution grid covering the Bay of Biscay and the English Channel was nested. Significant wave heights $H_{s}$, peak periods $T_{p}$ and mean directions $D_{\text {ir }}$ show large interannual variability, particularly when winter means are considered. A regression analysis on theses parameters over the period 1953-2009 show: (1) an increase in $\mathrm{H}_{\mathrm{s}}$ reaching + $0.01 \mathrm{~m} \mathrm{yr}^{-1}$ to the north of the study area; (2) an increase in Tp reaching $+0.015 \mathrm{~s} \mathrm{yr}^{-1}$ to the South of the Bay of Biscay. The comparison between time series of these 
parameters and winter means of the North Atlantic Oscillation (NAO) index shows: (1) a positive correlation between the NAO index and $\mathrm{H}_{\mathrm{s}}$, increasing northward of the study area; (2) a positive correlation between the NAO index and $\mathrm{T}_{\mathrm{p}}$, also increasing northward of the study area and (3) a weak correlation between the NAO index and $\mathrm{D}_{\text {ir }}$. The long-term trends for the studied wave parameters are finally explained by an increase in the NAO index over the second half of the $\mathrm{XX}^{\text {th }}$ century.

\section{Keywords:}

Wave climate - Numerical modelling - North Atlantic Oscillation

\section{Introduction}

En se propageant à la surface des océans, la houle transporte l'énergie accumulée lors des tempêtes et la dissipe à travers de nombreux processus, dont la compréhension et la quantification est primordiale pour de nombreux problèmes environnementaux et d'ingénierie. Par exemple, dans les zones côtières, le déferlement des vagues induit des gradients de tension de radiation (LONGUET-HIGGINS \& STEWART, 1964) à l'origine de courants longitudinaux (longshore currents), de courants sagittaux (rip currents) et de courants de retour par le fond (untertows). Ces processus étant principalement contrôlés par la hauteur (ou l'énergie), la direction et la période (ou la longueur d'onde) des vagues, il apparaît essentiel de connaître les variabilités temporelle et spatiale de ces paramètres, d'autant plus que celles-ci sont susceptibles d'évoluer en réponse au changement climatique (IPCC, 2007). Malheureusement, les enregistrements de houle sont trop épars et discontinus pour permettre une étude raisonnable de cette variabilité. Ce constat est valable dans le Golfe de Gascogne où, malgré les nombreux usages de ses eaux et de ses littoraux, les enregistrements continus de houle ne sont disponibles que depuis une vingtaine d'années, ce qui est trop court pour caractériser d'éventuelles cyclicités sur le long terme. Pour résoudre ce problème, BENOIT et al. (2006) ont réalisé une base de données d'état de mer le long des côtes Françaises sur la période 1979-2002 à partir de simulations numériques rétrospectives. A partir de cette base de données, LAFON et BENOIT (2006) ont estimé les conditions d'agitation extrêmes dans le Golfe de Gascogne et en Manche. Notre étude vise à étudier la variabilité de la houle dans le Golfe de Gascogne au cours des six dernières décennies grâce à des simulations numériques. Cette étude est basée sur les récents travaux de DODET et al. (2010) et son originalité tient à l'utilisation d'une stratégie de modélisation qui permet d'améliorer sensiblement les prédictions et d'étudier la variabilité de la houle à haute résolution dans le Golfe de Gascogne et la partie occidentale de la Manche. La seconde section de l'article décrit l'implémentation du modèle de vague dans le Golfe de Gascogne et sa validation. La troisième section décrit la variabilité de la houle dans le Golfe de Gascogne. Enfin la dernière section discute de l'origine de la variabilité mise en évidence et conclut sur les implications des résultats de cette étude. 


\section{Le modèle de vagues pour le Golfe de Gascogne}

\subsection{Description du modèle}

Le modèle utilisé dans notre étude est basé sur la version 3.14 du code WaveWatch III (WW3) (TOLMAN, 2009) et utilise la paramétrisation des termes source décrite par ARDHUIN et al. (2009a) et validée par ARDHUIN et al. (2009b) à l'aide de données satellites. Une première grille couvre $1^{\prime}$ Atlantique Nord, de $80^{\circ} \mathrm{W}$ à $0^{\circ} \mathrm{W}$ et de $0^{\circ} \mathrm{N}$ à $70^{\circ} \mathrm{N}$ avec une résolution de $1^{\circ}$. Une seconde grille est emboîtée dans le Golfe de Gascogne avec une résolution de $0.2^{\circ}$ (figure 1). Le modèle est forcé par les vents réanalysés du National Center for Environmental Predictions américain (NCEP) disponibles toutes les $6 \mathrm{~h}$ pour la période 1953-2009 (KALNAY et al., 1996). Une correction de $+3 \%$ a été appliquée au module de la vitesse des vents afin de corriger le léger biais en $\mathrm{H}_{\mathrm{s}}$ des résultats de DODET et al. (2010). Une simulation de 57 ans a été réalisée, afin d'obtenir des séries temporelles de $H_{s}, D_{i r}$ et $T_{p}$ espacées de $6 \mathrm{~h}$ et pour tous les nœuds de la seconde grille. Les simulations ont été réalisées pour un niveau moyen de la mer et les interactions avec les courants n'ont pas été prises en compte. Le problème du temps de calcul très élevé associé à cette simulation ( 2 mois de calcul sur un processeur performant) a été résolu en utilisant la version parallèle (MPI) de WW3 sur un Cluster Fujitsu-Siemens de 268 processeurs.

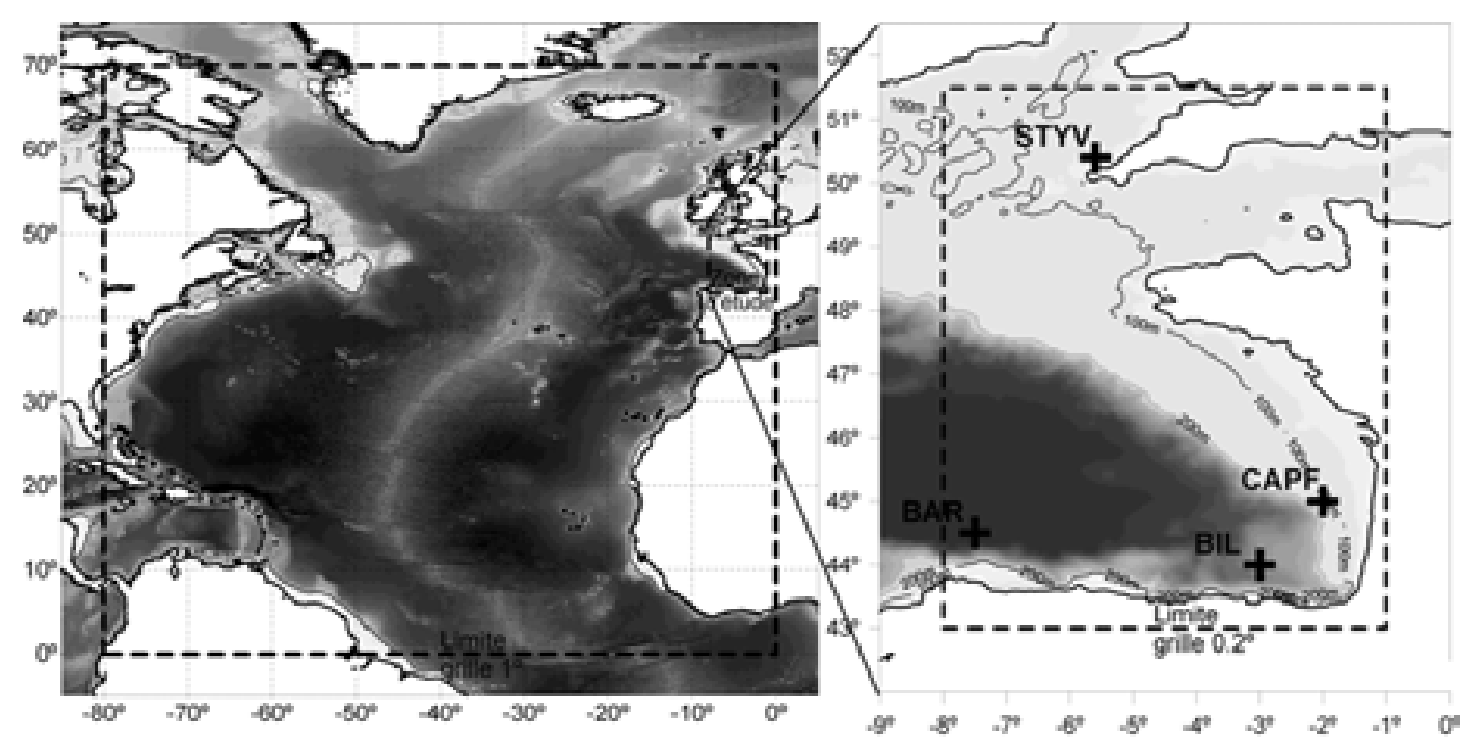

Figure 1. Carte bathymétrique de l'Atlantique Nord (grille 1) et du Golfe de Gascogne (grille 2), extension des grilles de calcul et positions des bouées utilisées pour la validation du modèle.

\subsection{Validation du modèle}

Les résultats du modèle ont été comparés aux mesures issues des bouées de Saint-Yves (STYV, Cornouaille anglaise), Cap Ferret (CAPF), Bilbao (BIL) et Bares (BAR), 
disponibles entre 1989 et 2009 (figure 1). Pour chacune des bouées, les bais, erreurs quadratiques moyennes (EQM) et erreurs quadratiques moyennes normalisées (EQMN) ont été calculés dans un premier temps par intervalles de $6 \mathrm{~h}$ (tableau 1). Le modèle reproduit les hauteurs significatives avec un biais inférieur à $0.1 \mathrm{~m}$ mais sans tendance claire et des EQM de l'ordre de $0.4 \mathrm{~m}$, ce qui correspond à environ $20 \%$ en EQMN. Les directions moyennes du modèle ne présentent pas de biais significatifs et les EQM varient de 12 à $30^{\circ}$ selon les sites. Enfin les périodes de pic sont reproduites avec un léger biais positif (environ 0.8 s) et une EQM d'environ 2 s, soit environ $20 \%$ en EQMN. Le niveau de précision du modèle est légèrement supérieur à celui obtenu par DODET et al. (2010), du fait de la correction du biais en Hs, mais aussi de l'emploi d'une grille à plus haute résolution dans le Golfe de Gascogne. Cependant, les erreurs observées sont du même ordre de grandeur que les tendances à long terme mises en évidence par plusieurs auteurs (BACON \& CARTER, 1993 ; BAUER, 2001). Sachant que cette étude s'intéresse aux moyennes hivernales des paramètres de la houle, ces erreurs ont aussi été calculées sur 1 mois. Cette procédure permet de diviser les EQM et EQMN par 2 à 3, avec des valeurs qui tendent vers le biais (tableau 1).

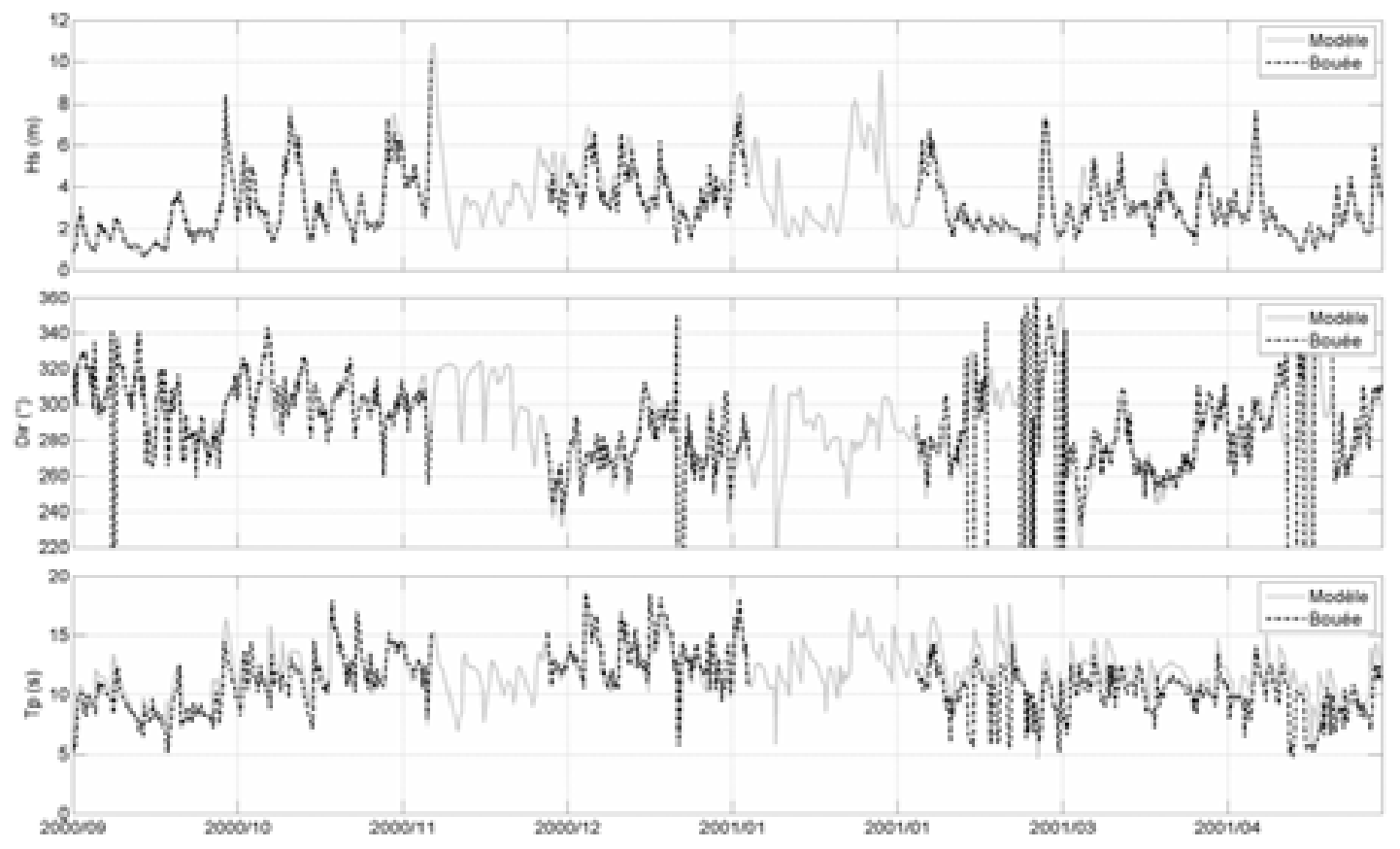

Figure 2. Comparaison entre les prédictions du modèle et la bouée de Bares (Espagne) en 2000 et 2001 
Tableau 1. Erreurs statistiques entre les données de vague issues du modèle et celles mesurés aux bouées de Saint-Yves (STYV, Cornouaille anglaise), Cap Ferret (CAPF), Bilbao (BIL) et Bares (BAR) pour les paramètres Hs, Dir et Tp. L'indication nc désigne les valeurs non calculées.

\begin{tabular}{|c|c|c|c|c|c|c|c|c|c|}
\hline \multirow[t]{2}{*}{ Bouée } & \multirow[t]{2}{*}{$\begin{array}{l}\text { Para- } \\
\text { mètre }\end{array}$} & \multicolumn{2}{|c|}{ Nombre de valeurs } & \multicolumn{2}{|l|}{ Biais } & \multicolumn{2}{|l|}{$E Q M$} & \multicolumn{2}{|l|}{$E Q M N$} \\
\hline & & $6 h$ & 1 mois & $6 h$ & 1 mois & $6 h$ & 1 mois & $6 h$ & 1 mois \\
\hline \multirow{3}{*}{$S T Y V$} & $H s$ & 686 & 11 & $0.1 \mathrm{~m}$ & $0.09 \mathrm{~m}$ & $0.38 m$ & $0.19 \mathrm{~m}$ & $17.03 \%$ & $11 \%$ \\
\hline & Dir & 686 & 11 & -2.4 & $3.5^{\circ}$ & $20.2^{\circ}$ & $4.92^{\circ}$ & $n c$ & $n c$ \\
\hline & $T p$ & 686 & 11 & $0.72 s$ & $0.8 s$ & $1.94 \mathrm{~s}$ & $0.89 s$ & $17.20 \%$ & $8.00 \%$ \\
\hline \multirow{3}{*}{$C A P F$} & $H s$ & 1166 & 6 & $0.1 \mathrm{~m}$ & $0.06 \mathrm{~m}$ & $0.4 m$ & $0.22 \mathrm{~m}$ & $22.00 \%$ & $11 \%$ \\
\hline & Dir & 1166 & 6 & $1.54^{\circ}$ & $2.6^{o}$ & $12.7^{\circ}$ & $7.2^{\circ}$ & $n c$ & $n c$ \\
\hline & $T p$ & $n c$ & $n c$ & $n c$ & $n c$ & $n c$ & $n c$ & $n c$ & $n c$ \\
\hline \multirow{3}{*}{$B I L$} & $H s$ & 17320 & 97 & -0.1 & -0.11 & $0.46 m$ & $0.27 \mathrm{~m}$ & $24.00 \%$ & $13.00 \%$ \\
\hline & Dir & 17320 & 97 & -3.18 & -3.67 & $22.35^{\circ}$ & $8.76^{\circ}$ & $n c$ & $n c$ \\
\hline & $T p$ & 17320 & 97 & $0.95 s$ & $0.97 s$ & $2.1 \mathrm{~s}$ & $0.78 s$ & $19.20 \%$ & $7.10 \%$ \\
\hline \multirow{3}{*}{$B A R$} & $H s$ & 12863 & 75 & -0.002 & -0.05 & $0.48 m$ & $0.25 \mathrm{~m}$ & $0.19 \%$ & $10 \%$ \\
\hline & Dir & 12863 & 75 & -4.7 & -2.6 & $34^{\circ}$ & $12^{\circ}$ & $n c$ & $n c$ \\
\hline & $T p$ & 12863 & 75 & $0.80 s$ & 0.6 & $2.15 \mathrm{~s}$ & $1.1 \mathrm{~s}$ & $19 \%$ & $6.50 \%$ \\
\hline
\end{tabular}

\section{Variabilité de la houle dans le Golfe de Gascogne}

\subsection{Moyennes hivernales et variabilité}

Les séries temporelles des différents paramètres des vagues ont été archivées pour tous les nœuds de la grille 2 (figure 1) entre 1953 et 2009 et avec un pas de temps de $6 \mathrm{~h}$. Les moyennes hivernales (entre le 1er décembre et le 31 mars), leur écart-type ainsi que leur tendance linéaire sur la période d'étude ont été calculés et sont montrés sur la figure 3. Les $H_{\mathrm{s}}$ moyennes diminuent vers l'est, de $3.5 \mathrm{~m}$ à l'entrée du Golfe de Gascogne à moins de $2 \mathrm{~m}$ dans la Manche. Les écarts-types associés diminuent de $0.5 \mathrm{~m}$ à l'ouest à $0.2 \mathrm{~m}$ à l'est, si bien que leur valeur normalisée par la moyenne est assez homogène sur l'ensemble de la zone (environ 15\%). Les directions moyennes varient de $260^{\circ}$ au nord de la zone à $300^{\circ}$ au sud, avec un écart-type variant de $9^{\circ}$ à l'entrée du Golfe de Gascogne à $4^{\circ}$ au sud-ouest de la zone. Une variabilité beaucoup plus forte est observée le long des côtes de la Manche, avec des écarts-types qui atteignent localement $20^{\circ}$.

Enfin les périodes de pic varient de $11 \mathrm{~s}$ au nord de la zone à $12 \mathrm{~s}$ au sud, avec des valeurs plus faibles le long des côtes de la Manche (7-8 s). Les écarts-type associés sont homogènes et de l'ordre de 0.7 s sur l'ensemble du Golfe de Gascogne, avec des valeurs plus fortes dans le Golfe Normo-Breton et les côtes sud de Bretagne. 

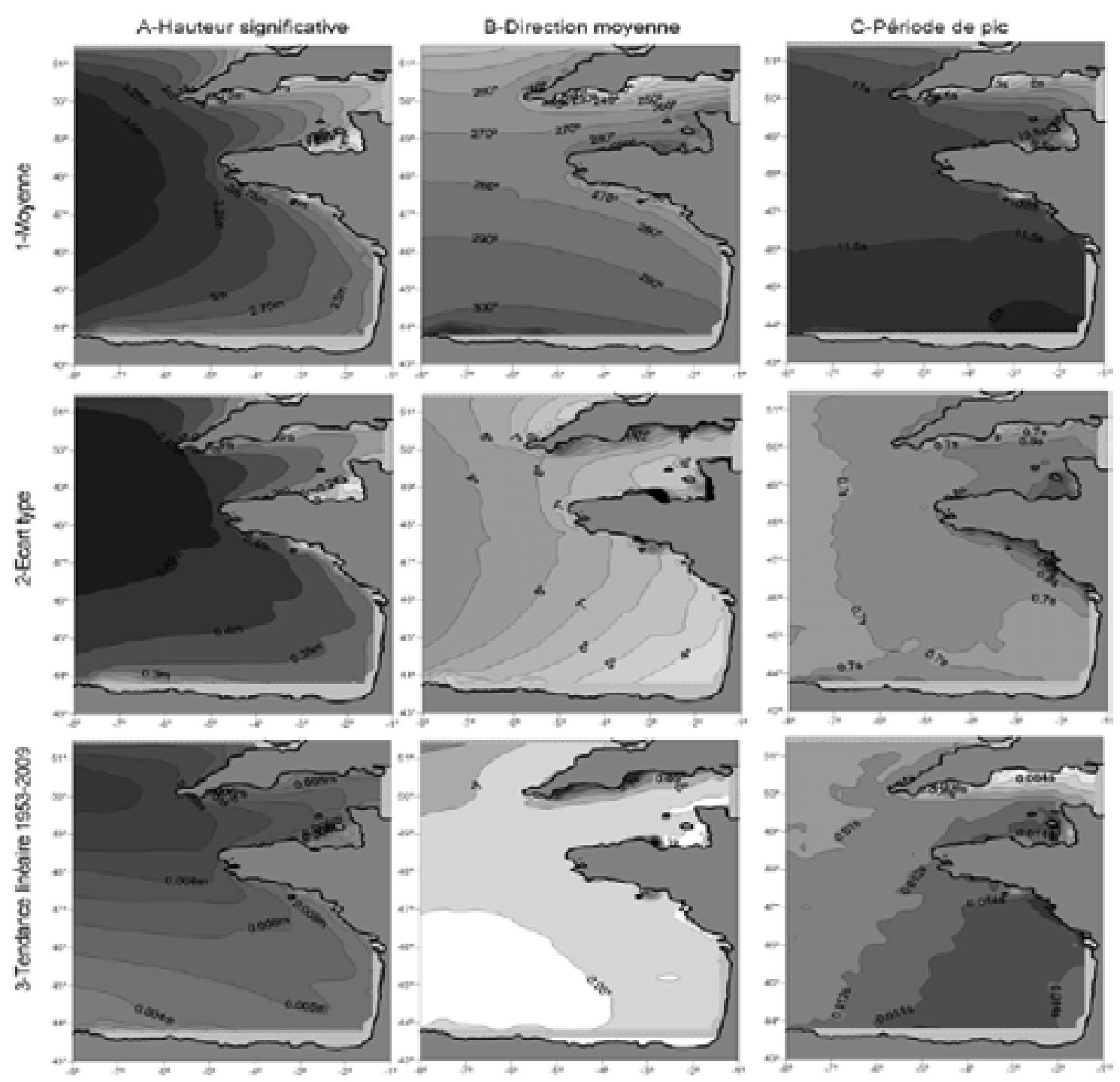

Figure 3. Moyennes hivernales (ligne 1), écarts types (ligne 2) et tendance linéaire sur la période 1953-2009 (ligne 3) pour les hauteurs significatives (colonne 1), directions moyennes (colonne 2) et périodes de pic (colonne 3).

\subsection{Tendances à long-terme}

Pour chaque nœud du maillage, une régression linéaire a été effectuée sur les moyennes hivernales de la période 1953-2009 afin d'étudier les tendances à long terme (figure 3). Compte tenu des erreurs associées au modèle présentées dans le tableau 1, seules les tendances significatives sont décrites dans ce paragraphe. Ainsi, sur la période 19532009, les $\mathrm{H}_{\mathrm{s}}$ augmentent significativement sur l'ensemble de la zone, avec des valeurs atteignant $+0.6 \mathrm{~m}$ au large de la Cornouaille Anglaise $\left(\right.$ soit $+0.01 \mathrm{~m} \mathrm{an}^{-1}$ ). Sur la même période, les directions moyennes ne présentent pas de tendances significatives, excepté le long des côtes anglaises de la Manche, où elles subissent une rotation horaire atteignant localement $10^{\circ}$. Enfin les Tp augmentent sur l'ensemble de la zone, avec un 
maximum au sud-ouest du Golfe de Gascogne où cette augmentation atteint $1 \mathrm{~s}$ sur la période 1953-2009.

\section{Discussion et conclusions}

\subsection{Corrélation avec l'oscillation Nord-Atlantique}

L'Oscillation Nord Atlantique (NAO) a été décrite en détail par HURREL (1995) et l'indice qui la caractérise est basé sur la différence entre les moyennes mensuelles de la pression atmosphérique normalisée à Reykjavik (Islande) et à Ponta Delgada (Açores, Portugal). Un indice positif (NAO+) correspond à une situation où un anticyclone puissant est centré sur les Açores et où des dépressions creuses circulent d'ouest en est au niveau de l'Islande. A l'inverse, un indice négatif (NAO-) correspond à un anticyclone faible et des dépressions moins creuses et dont la trajectoire est plus variable. Plusieurs auteurs ont déjà montré que la variabilité en $\mathrm{H}_{\mathrm{s}}$ dans l'Atlantique Nord était contrôlée par l'oscillation Nord Atlantique (NAO) (BACON \& CARTER, 1993 ; BAUER, 2001 ; DODET et al., 2010). Afin de vérifier cette hypothèse dans le Golfe de Gascogne et de l'étendre à d'autres paramètres, les coefficients de corrélation entre les moyennes hivernales des paramètres des vagues et celles de l'indice NAO (HURREL, 1995) ont été calculés (figure 4). Les $\mathrm{H}_{\mathrm{s}}$ sont moyennement corrélées avec l'indice NAO dans le Golfe de Gascogne, avec des valeurs de l'ordre de 0.3 à 0.4. Des valeurs plus élevées (0.6) sont observées le long des côtes nord de Bretagne et de Cornouaille. Les directions sont faiblement corrélées avec l'indiceNAO dans le Golfe de Gascogne mais présentent une corrélation positive le long des côtes sud de Cornouaille et une corrélation négative le long des côtes nord-Bretonnes et Normandes et le long de la côte nord de Cornouaille. Enfin les périodes de pic présentent une corrélation significative avec l'indice NAO sur l'ensemble de la zone. Les situations de type NAO + sont associées à de forts gradients de pression atmosphérique qui génèrent de forts vents d'ouest à sud-ouest aux latitudes élevées de l'Atlantique Nord. Ces forts vents d'ouest génèrent des houles plus fortes et plus longues, ce qui explique les corrélations positives observées pour $\mathrm{H}_{\mathrm{s}}$ et $\mathrm{T}_{\mathrm{p}}$. Conformément aux résultats de DODET et al. (2010), les directions moyennes de la houle sont peu corrélées avec l'indice NAO dans le Golfe de Gascogne. Cette étude à plus haute résolution permet par contre de mettre en évidence deux zones de corrélation négatives le long des côtes sud de la Manche et le long de la côte nord de Cornouaille et une zone de corrélation positive au niveau de la côte sud de Cornouaille. La carte des directions moyennes (figure 3) montre que les houles effectuent une réfraction aux abords de la Manche, qui induit une rotation horaire (de $270^{\circ} \mathrm{N}$ vers $300^{\circ} \mathrm{N}$ ) au niveau des littoraux orientés au nord et antihoraire (de $270^{\circ} \mathrm{N}$ vers $230^{\circ} \mathrm{N}$ ) au niveau de ceux orientés au sud. Nous proposons que la corrélation négative entre l'indice NAO et la direction moyenne des vagues observée au niveau des littoraux orientés au nord traduise une dominance des mers de vent d'ouest à sud-ouest 
pendant les hivers de situation NAO+. A l'inverse, nous proposons que cette dominance des mers de vent d'ouest à sud-ouest explique la corrélation positive observée le long des côtes sud de Cornouaille.
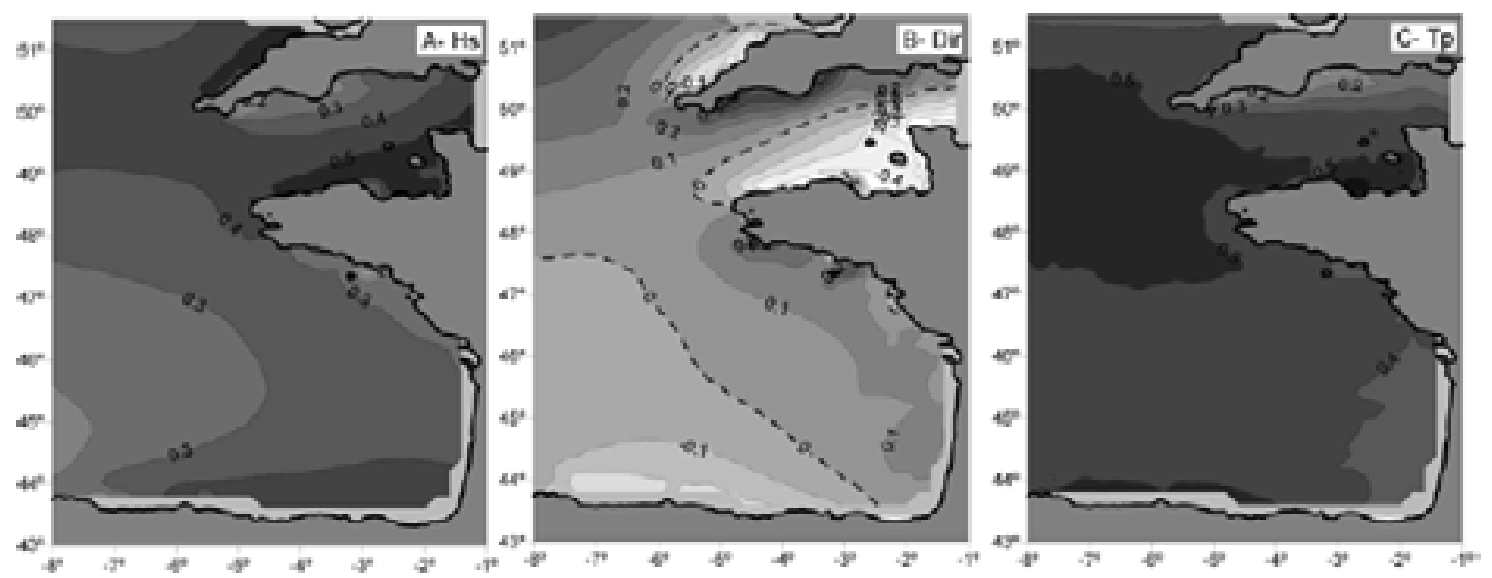

Figure 4. Cartes de coefficients de corrélation Pearson entre les moyennes hivernales de l'indice NAO et les $\mathrm{Hs}(A)$, les Dir $(B)$ et les Tp $(C)$.

\subsection{Origine des tendances à long terme}

Les cartes des tendances à long-terme (figure 3) et des coefficients de corrélation (figure 4) présentent un certain nombre de similitudes du point de vue des répartitions spatiales. Ainsi les zones caractérisées par une augmentation de $\mathrm{H}_{\mathrm{s}}$ et $\mathrm{T}_{\mathrm{p}}$ sont associées à une corrélation positive avec l'indice NAO. Le long de la côte sud de Cornouaille, la rotation horaire des directions moyennes est associée à une corrélation positive entre $D_{\text {ir }}$ et l'indice NAO. HURREL (1995) a montré que l'indice NAO avait fortement augmenté au cours de la seconde moitié du $X{ }^{\text {ème }}$ siècle et que les valeurs observées en 1989 étaient les plus fortes depuis 1864. Cette augmentation de l'indice NAO sur la période d'étude permet d'expliquer facilement les augmentations de $\mathrm{H}_{\mathrm{s}}$ et $\mathrm{T}_{\mathrm{p}}$ sur l'ensemble de la zone. Une autre conséquence de cette augmentation est la présence de vents d'ouest à sud-ouest plus forts aux latitudes élevées, qui peuvent localement induire des mers de vent dominant le régime d'agitation dans les zones qui ne sont pas directement exposées aux houles. Nous proposons que ce phénomène explique la rotation horaire de $10^{\circ}$ des directions moyennes observée le long de la côte sud de Cornouaille.

\subsection{Implications et conclusion}

Cette étude a montré que dans le Golfe de Gascogne et en Manche, les climats de vague hivernaux étaient soumis à une forte variabilité interannuelle. Sur la période 1953-2009, une augmentation importante des $\mathrm{H}_{\mathrm{s}}$ et $\mathrm{T}_{\mathrm{p}}$ a pu être mise en évidence et a été expliquée par une augmentation de l'indice NAO sur la période d'étude. L'érosion côtière qui 
frappe les littoraux atlantiques depuis plusieurs décennies est classiquement imputée à l'augmentation du niveau marin et à une diminution des apports sédimentaires continentaux. Pourtant, de nombreuses plages sont déconnectées des systèmes de drainage continentaux et subissent une érosion qui ne peut pas être expliquée par la seule augmentation du niveau marin. L'augmentation de $\mathrm{H}_{\mathrm{s}}$ mise en évidence dans cette étude (jusqu'à $+0.6 \mathrm{~m}$ ) pourrait expliquer cette érosion. Les résultats présentés dans cette étude ont probablement de nombreuses autres implications qu'il conviendrait d'étudier. A cette fin, les séries temporelles des paramètres de houle pourront être mises à disposition sur simple demande aux auteurs de l'article.

\section{Remerciements :}

Cette étude n'aurait pu être réalisée sans la disponibilité du code WW3 développé à la NOAA par H. Tolman ainsi que les données de vents ré-analysées du NCEP. Fabrice Ardhuin est chaleureusement remercié pour nous avoir communiqué régulièrement ses dernières paramétrisations de WW3. Les données de houle utilisées pour la validation ont été fournies par l'équipe METHYS (UMR EPOC, Université Bordeaux I), les ports d'Espagne et l'entreprise South West Rda. Guillaume Dodet est financé par le projet Européen MICORE (Contrat 202798).

\section{Références bibliographiques}

ARDHUIN F., CHAPRON B., COLLARD F. (2009a). Strong decay of steep swells observed across oceans. Geophysical Research Letters 36 (6), art. no. L06607. doi:10.1029/2008GL037030

ARDHUIN F., MARIE L., RASCLE N., FORGET P., ROLAND A., (2009b). Observation and estimation of Lagrangian, Stokes and Eulerian currents induced by wind and waves at the sea surface. Journal of Physical Oceanography 39 (11), pp 2820-2838. doi:10.1175/2009JPO4169.1

BACON S., CARTER D.J.T. (1993). A connection between mean wave height and atmospheric pressure gradient in the North Atlantic. International Journal of Climatology 13, pp 423-436. doi:10.1002/joc.3370130406

BAUER E. (2001). Interannual changes of the ocean wave variability in the North Atlantic and in the North Sea. Climate Research 18, pp 63-69. doi:10.3354/cr018063

BENOIT M., LAFON F., GOASGUEN G. (2006). Constitution et exploitation d'une base de données d'états de mer le long des côtes Françaises par simulation numérique sur 23 ans. Actes des IX ${ }^{\text {èmes }}$ Journées Nationales Génie Côtier Génie Civil, 12-14 septembre 2006, Brest (France), pp 21-30. doi:10.5150/jngcgc.2006.003-B

DODET G., BERTIN X., TABORDA R. (2010). Wave climate variability in the NorthEast Atlantic Ocean over the last six decades. Ocean Modelling 31, pp 120-131. doi:10.1016/j.ocemod.2009.10.010 
Thème 1 - Hydrodynamique côtière

HURREL J.W. (1995). Decadal trends in the North Atlantic Oscillation: regional temperatures and precipitations. Science 269, pp 676-679. doi:10.1126/science.269.5224.676 IPCC (2007). Climate change 2007: impacts, adaptation and vulnerability, Working Group I Report, The Physical Science Basis. Chapter 10, Global Climate projection, World Meteorological Organization, Genève, 2007, pp 812-822.

LAFON F., BENOIT M. (2006). Estimation of extreme wave conditions from hindcast simulations with application to the wave climate along the French coasts. Proceedings of the $30^{\text {th }}$ International Conference on Coastal Engineering, 3-8 September 2006, San Diego (USA), pp 739-751.

KALNAY E., KANAMITSU M., KISTLER R., COLLINS W., DEAVEN D., GANDIN L., IREDELL M., SAHA S., WHITE G., WOOLEN J., ZHU Y., CHELLIAH M., EBISUZAKI W., HIGGINS W., JANOVIAC J., MO K.C., ROPELEVSKI C., WANG J., LEETMAA A., REYNOLDS R., ROY JENNE R., JOSEPH D. (1996). The NCEP/NCAR 40-year reanalysis project. Bull. American Met. Soc. 77, pp 437-470. doi:10.1175/1520-0477(1996)077<0437:TNYRP>2.0.CO;2

LONGUET-HIGGINS M.S., STEWART R.W. (1964). Radiation stresses in water waves: a physical discussion, with applications. Deep-Sea Research 11, pp 529-562.

TOLMAN H.L. (2009). User manual and system documentation of WAVEWATCH III version 3.14. NOAA/NWS/NCEP/MMAB Technical Note 276, 194 p. 